\title{
Context Reinstatement
}

\author{
Jeremy R. Manning, Ph.D. \\ Affiliation: Department of Psychological and Brain Sciences \\ Dartmouth College \\ Hanover, NH 03755 \\ Email: jeremy.r.manning@ dartmouth.edu
}

August 10, 2020

\begin{abstract}
Context reinstatement is the process by which we incorporate thoughts from our past into our current mental state. This chapter is concerned with characterizing the cognitive and neuropsychological underpinnings of context reinstatement. Modern theories of context reinstatement are inextricably tied to theories of how we process and perceive the present. The primary goal of this chapter is to present a conceptual framework for characterizing how experiences unfold in time, and how our mental states at each moment relate to our experiences. This framework allows us to compare, contrast, and test different theories of context reinstatement. A second component of this chapter is concerned with two fundamental properties of how our experiences unfold, and how we perceive and remember them: scale invariance and event segmentation. The chapter concludes with a reflection on the proposed role of context reinstatement in facilitating a range of important memory-related cognitive functions.
\end{abstract}

Keywords: Episodic memory, context reinstatement, autocorrelation, scale invariance, event boundary 


\section{Overview}

This chapter is concerned with how the mental context in which we experience each new moment affects how we learn and remember. Essentially, contextual information serves as a sort of mental bookmarking system that allows us to tag each moment of our experience with a unique identifier, and to organize related experiences. We will begin by exploring what it means to remember-i.e., to mentally revisit our past experiences.

\section{Where do we "go" when we remember?}

The blend of thoughts we carry with us into each new moment provides a context for interpreting new experiences, cataloging those new experiences in our memories, bringing our related prior experiences to bear on the problem of deciding how to behave, and generating expectations and predictions about the future. Whereas our immediate sensory experiences (what we are seeing, hearing, touching, tasting, and smelling) reflect measurements of the external world (i.e., the world outside of our brain), contextual thoughts are only indirectly related to the physical goings-on of each new moment. Although contextual thoughts may be directly influenced by the external world, they may also be colored by prior external experiences (e.g., what happened in other moments prior to now) or by our internal states (e.g., our goals, emotions, metabolic needs, physiology, high-level understanding of our current situation, etc.) in ways that are only indirectly tied to our immediate sensory experiences. Because they are not directly locked to our moment-by-moment sensory experiences, contextual thoughts often change more gradually than our sensory experiences of the external world. Different contextual thoughts can drift at different rates, or persist for different amounts of time. For example, our understanding of a given spoken word may require integrating over auditory information acquired over less than a second; understanding a sentence in which the word is embedded might entail integrating over auditory information acquired over several seconds; and understanding the broader conversation might require integrating over many minutes. 
Each type of information being integrated at a given timescale (phonemes within a word, words within a sentence, sentences within a conversation, etc.) provides a "context" for interpreting other information being integrated over shorter timescales. Modern theories of episodic memory posit that more slowly drifting thoughts contextualize faster-drifting thoughts (e.g., see review by Manning et al., 2015).

\section{The autobiographical timeline and context reinstatement}

Each moment of our subjective experience is characterized by a unique blend of externally and internally driven thoughts (Heraclitus, BCE). If remembering entails reactivating thoughts from our past, we can ask: at the moment of remembering, how similar is our current blend of thoughts to the thoughts we had at each prior moment? Effectively, we are spreading our current mental state back through our past, revisiting each prior experience to some quantifiable extent (Fig. 1). This is analogous to a quantum wave function spreading its probability mass over space (Manning, 2019). The shape of this distribution can tell us about how we mentally revisit our past when we remember. For example, do our thoughts at the time of remembering incorporate information from a continuous blend of timepoints from our past? Or just a single timepoint (or range of timepoints)? Or a discrete set of timepoints (or ranges)? Each of these possibilities encompasses different hypotheses about context reinstatement and memory. Understanding how we might begin to explore or distinguish between these hypotheses requires first examining how our experiences unfold over time.

\section{How do experiences unfold over time?}

The physical world, though highly complex, is also highly constrained at the macro level by wellstructured and (often) predictable rules. Our sensory, attentional, and memory systems are tuned to exploit these regularities to efficiently represent the external world, fill in ambiguities in our ongoing or remembered experiences, and generate predictions. For example, the components of 


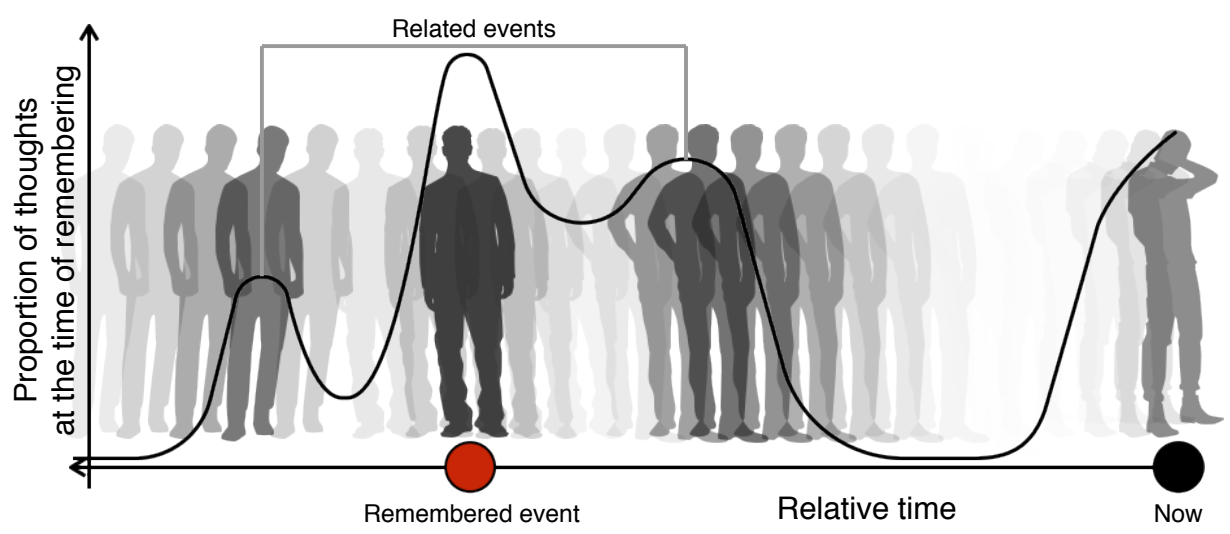

Figure 1: Where do we "go" when we remember? When we remember a past event, our thoughts (at the time of remembering) reflect a weighted blend of our thoughts just prior to now (black dot), thoughts we had around the time we experienced the remembered event (red dot), and thoughts we had during other related experiences or events. We can therefore ask: to what extent are thoughts related to each moment from our past reflected in our current thoughts?

objects occupy contiguous points in space, so knowing that an object is present at one point in space provides a predictive signal about whether that object also occupies an immediately adjacent point in space, even if that adjacent point is visually occluded. Similarly, our body's position in space varies continuously (i.e., we cannot teleport between non-adjacent spatial locations). This means that our spatial position at one moment tends to be similar to our spatial position at the next moment. In general, our physical environment is highly autocorrelated: all else equal, our experiences tend to progress smoothly and gradually along any given dimension (temporal, spatial, situational, conceptual, etc.). The autocorrelations in our experiences are also scale invariant in that they exhibit similar structure at a wide range of scales. For example, just as our sensory experience tends to be similar from one moment to the next (on the scale of milliseconds to seconds), general properties of our sensory environment, such as the room we are in, tend to change gradually- but over longer time scales (minutes to hours). Higher order features of our experience such as the weather (hours to days), season (months), or architectural features of the town or city we live in (years to decades) change at still longer timescales. Analogous principles hold for abstract (non-sensory) features of the external environment, such as social or situational interactions, 
and for internally driven thoughts such as our goals and emotions.

Our brains themselves also exhibit autocorrelated activity. In the extreme this is trivially true; our brains are biophysical systems that cannot change infinitely quickly, so neural activity patterns are necessarily similar (to some extent) from one moment to the next. However, autocorrelated activity patterns at the time scales we typically examine in laboratory memory studies are far stronger and persist for far longer than is strictly necessary from a biophysical standpoint. For example, transient changes in neuronal membrane potentials during action potentials can last just a few milliseconds (Hodgkin and Huxley, 1952) and even local field potentials that reflect the synchronized activities of tens of thousands of neurons can exhibit millisecond timescale changes (Buzsáki, 2006; Fries, 2005). Nevertheless, our brain activity pattern can remain reliably autocorrelated at timescales of seconds or longer- and these long timescale autocorrelations predict how our ongoing experiences are remembered later (e.g., Folkerts et al., 2018; Howard et al., 2012; Manning et al., 2011; Manns et al., 2007).

One consequence of autocorrelations in our physical and neuropsychological experiences is that it is often difficult to pin down the precise moment in time that a thought (or its associated brain activity pattern) occurred. If a thought is driven by some aspect of our external environment, then because our experience changes gradually, the same factors that triggered the thought are likely to be present across successive moments as they fade into and out of our immediate sensory experience. Further, as we will discuss below, our memory systems temporally blur our ongoing experiences at a range of timescales. This means that the subjective effects of an experience tend to linger even after the external cues are no longer present. If we were to measure how much our thoughts at the time of remembering reflected the thoughts we had at different moments from our past (e.g., as in Fig. 1), the presence of autocorrelations in our moment-by-moment experiences imply that the "match" to one moment's thoughts will be similar to the match to temporally proximal other moments' thoughts. This temporal blurring arises from two sources that are often difficult to separate: autocorrelations in our experiences themselves, and additional autocorrelations induced 


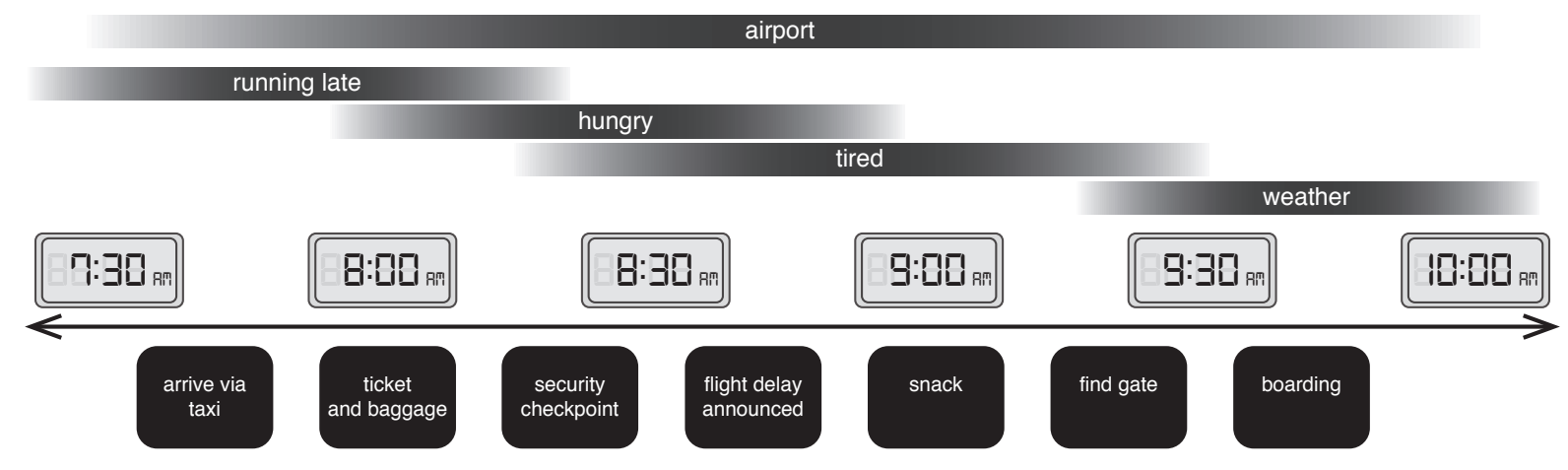

Figure 2: Contextual thoughts drifting at a range of timescales. The timeline displays a hypothetical sequence of thoughts during a harried late arrival at the airport. The labels at the bottom of the figure denote a series of events, experienced between arriving at the airport and boarding an airplane. In addition to thoughts about the moment-by-moment experiences during each event (not shown), higher-order thoughts driven both by external and internal experiences often persist across multiple events. These persistent thoughts serve as a context for faster-drifting lower-order thoughts (e.g., about one's immediate moment-by-moment experiences), and are themselves contextualized by even more slowly drifting thoughts (e.g., a general sense of being at the airport).

by our memory systems that temporally blur our ongoing experiences (Fig. 2).

Although autocorrelations are perhaps the dominant organizing principle of our unfolding experiences, occasionally we can experience more abrupt transitions, termed event boundaries. Conceptually, event boundaries occur when we transition from one situation or experience to another different situation or experience. For example, if you are speaking with a friend at a party, the beginning and end of your conversation would constitute event boundaries. Just as ongoing experiences exhibit structure at a range of scales, event boundaries also occur at a range of scales. For example, event boundaries may occur on the order of seconds or minutes (e.g., within a single conversation that covers different content), minutes to hours (e.g., as you converse with different individuals at the same party), or longer (e.g., heading to the party, attending the party, and then heading home). Event boundaries constitute an important special case of how our experiences unfold, because they break the typically autocorrelated structure of our ongoing experience. In other words, whereas our moment-by-moment experiences within an event tend to be strongly au- 
tocorrelated, our experience changes much more abruptly across event boundaries. This provides a potential means of separating out the sources of autocorrelations in our thoughts: across event boundaries, our experiences themselves may change abruptly even if our ongoing thoughts (and neural activity patterns) related to those experiences exhibit lingering traces of the pre-boundary event. In addition to temporal event boundaries, analogous boundaries pervade our experiences in other domains including object features and boundaries, environmental boundaries, semantic concepts, social constructs, etc. Like temporal event boundaries, boundaries in other domains also occur at a range of scales. The interplay between autocorrelations and boundaries serves as a key organizing principle for our memory systems. For example, this enables us to segment our ongoing experiences along our autobiographical timeline into discrete events that we can efficiently catalogue, retrieve, and communicate to others (e.g., Zadbood et al., 2017). This interplay also allows us to form effective predictions about our current situation and adapt our behaviors and expectations accordingly (e.g., Ranganath and Ritchey, 2012).

\section{How do we revisit our past experiences?}

Tulving (1983) characterized episodic memory as "mental time travel," whereby we mentally revisit the moment of our past when we experienced the remembered event. Modern theories of context reinstatement (e.g., Diana et al., 2007; Howard and Kahana, 2002; Howard et al., 2014; Manning, 2019; Polyn et al., 2009; Ranganath, 2018; Sederberg et al., 2008; Shankar and Howard, 2010, 2012; Shankar et al., 2009) have clarified and expanded on this view. These models were inspired in part by earlier theoretical work suggesting that temporally proximal experiences become linked through their shared contextual features (Atkinson and Shiffrin, 1968; Estes, 1955). The emerging insight from this body of work is that our brains maintain parallel representations of our ongoing (internal and external) experiences that are each temporally blurred with a different time constant. Conceptually, higher-level thoughts (e.g., about the broader environment, conceptual space, situation, or emotional state) reflect experiences that are blurred with longer time constants, 
whereas lower-level thoughts (e.g., about the details of the environment, conceptual space, situation, or emotional state) tend to change more rapidly. Each of these representations is maintained by a neural integrator that responds at a different timescale. These integrators can be thought of as individual neurons, small networks of neurons, entire brain regions, or combinations thereof. Further, an individual integrator might respond to one or more aspects of experience according to its receptive field (e.g., the perceived luminance of some part of the visual field of view; the estimated friendliness of a perceived face; the degree to which cats are present in one's immediate surroundings; etc.). As we will see later in this chapter, there are neurons in the hippocampus, and networks of brain regions distributed throughout cortex, that act like neural integrators by preferentially responding to experiences that unfold at specific timescales (Aly et al., 2018; Hasson et al., 2008; Honey et al., 2012a; Lerner et al., 2011; MacDonald et al., 2011; Mau et al., 2018; Pastalkova et al., 2008).

If an integrator responds (to a particular feature) with a time constant $\rho$, then we can use the function $y(t, \rho)$ to describe its activity at time $t$ :

$$
y(t, \rho)=\rho y(t-1, \rho)+(1-\rho) f(t)
$$

where $f(t)$ represents the value at time $t$ of the feature the integrator is responding to. In other words, the integrator's response at time $t$ is a weighted blend of its response in the previous timepoint (i.e., $y(t-1, \rho))$ and the feature's value at time $t$ (i.e., $f(t)$ ). When the weight $(\rho)$ is larger, the integrator's responses are more "temporally sticky" in that its activity patterns will change more gradually. This is because its responses will be driven more by its own activity in the previous moment than by the perceived feature(s) it is responding to.

Modern theories of contextual drift and reinstatement posit that our brains maintain a family of these neural integrators, each with its own time constant (value of $\rho$ ) and preferred stimulus feature(s). Conceptually, this means that we maintain parallel representations of our ongoing ex- 
periences, where each representation reflects information that is changing at a different timescale. These parallel representations become associated, enabling us to retrieve information about our prior experiences (and other related or temporally nearby experiences). Shankar and Howard (2012) showed that, if the time constants of these integrators are known, it is possible to use the activity of a population of neural integrators to efficiently reconstruct a remembered time course of the past, using a mathematical operation called the inverse Laplace transform (see Box for details).

\section{Box: Using the inverse Laplace transform to simulate memory retrieval}

The Fourier Transform takes a function of $t$ (e.g., of time, space, etc.), $f(t)$, and creates a new function of frequency, $F(\omega)$ :

$$
\begin{aligned}
F(\omega) & =\int_{-\infty}^{\infty} f(t) e^{-i \omega t} d t=\int_{-\infty}^{\infty} f(t) \cos (\omega t)-i \sin (\omega t) d t \\
& =\int_{-\infty}^{\infty} f(t) \cos (\omega t) d t-i \int_{-\infty}^{\infty} f(t) \sin (\omega t) d t
\end{aligned}
$$

Note that Equation 2 uses Euler's formula to re-write the exponential term as a sum of sine and cosine terms. Intuitively, Equation 3 asks: how much does a cosine wave at a given frequency, $\omega$, contribute to the original function, $f(t)$ ? The Fourier Theorem states that (nearly) any function may be represented as the weighted sum of cosine functions at different frequencies. Equation 3 tells us how to find the specific weights for each frequency. When we evaluate the integral at the frequency $\omega$, the result will be a complex number whose magnitude tells us how much $f(t)$ "looks like" a cosine function with a frequency of $\omega$. (The complex angle tells us the phase offset of that frequency's cosine function.) Further, $F(\omega)$ is invertible, such that we can uniquely recover the original $f(t)$ as long as we know $F(\omega)$.

The Laplace Transform is an extension of the Fourier Transform that takes $f(t)$ and 
creates a new function of decay and frequency, $F(s)$ :

$$
\begin{aligned}
F(s) & =\int_{0}^{\infty} f(t) e^{-s t} d t=\int_{0}^{\infty} f(t) e^{-(\alpha+i \omega) t} d t \\
& =\int_{0}^{\infty} f(t) e^{-i \omega t} e^{-\alpha t} d t
\end{aligned}
$$

The key difference between the Fourier Transform and Laplace Transform is the addition of the $e^{-\alpha t}$ term in Equation 5. Just as the Fourier Transform decomposes $f(t)$ into a weighted sum of cosines at different frequencies, the Laplace Transform decomposes $f(t)$ into a weighted sum of decaying cosines at different frequencies.

Post (1930) showed that we can approximate the Laplace Transform of $f(t)$ using a set of integrators like those defined in Equation 1, each with a different time constant ( $\rho$, where $\rho \propto$ $s^{-1}$ ). We can use the activations of the set of integrators at timepoint $t$ as an approximation of $F(s)$, evaluated at the corresponding values of $s$ for each integrator. Further, this operation is invertible: we can recover an approximation of the original $f(t)$ that the integrators were responding to, using only this approximation of $F(s)$ :

$$
\hat{f}(t, \hat{t})=\frac{(-1)^{k}}{k !} \rho^{k+1} y^{(k)}(t, \rho)
$$

where $k$ is a parameter that determines the precision of the reconstruction (where accuracy improves as $k \rightarrow \infty)$ and where $y^{(k)}(t, \rho)$ is the $k^{\text {th }}$ derivative of $y(t, \rho)$ evaluated at time $t$. Here $t$ represents the experienced time, whereas $\hat{t}$ represents the remembered times. As the number of integrators and the range of time constants grows, our ability to recover $f(t)$ using the inverse Laplace transform improves.

This set of mathematical principles has profound implications for how our memory systems might work. In particular, these principles demonstrate that it is possible to reconstruct 
a detailed history of an experience using only one moment's snapshot of the activity patterns of a set of neural integrators. Further, in leveraging the approximate inverse Laplace transform to reconstruct the past, Shankar and Howard (2012)'s model makes a number of important testable predictions about how we mentally revisit that past. For example, given a fixed set of integrators, the recovery of $f(t)$ is most accurate for recent timepoints, and becomes progressively worse as one attempts to recover $f(t)$ at earlier and earlier timepoints (Fig. 3).

Another interesting feature of this approach relates to the specific temporal markers of our experiences versus of our memories of those experiences. Whereas our experiences are perceived with respect to each moment $t$, Equation 6 suggests that our memories are catalogued with respect to a potentially different sequence of moments, $\hat{t}$. Characterizing potential discrepancies between $t$ and $\hat{t}$ can allow us to study how our experiences are distorted (temporally) when filtered through the lens of our memories of those experiences.

Figure 3 illustrates a sample time course predicted by Shankar and Howard (2012)'s model. The black lines in each panel show the time courses, $f(t)$, of a single "feature" (i.e., some aspect of ongoing experience). Each of the colored lines reflects one of $N=50$ temporally blurred version of $f(t)$, using a different time constant $\rho$ (Eqn. 1). The gray lines in each panel reflect a reconstructed time course, $\hat{f}(t, \hat{t})$, using the vector of $N$ temporally blurred signals evaluated at $y(t=10)$ (i.e., the activities of each neural integrator at time $t$ ), for $\hat{t}=10^{\frac{1}{N}^{x}}, x \in\{1, \ldots, N\}$. The reconstructions are computed using Post (1930)'s approximation for the inverse Laplace transform (Eqn. 6).

The Shankar and Howard (2012) model provides one example of a context reinstatement account of memory that incorporates most current theories about how we represent and revisit our ongoing experiences. In general, context reinstatement theories of memory make several distinct testable claims about how we remember. The four common tennets of these theories are: 

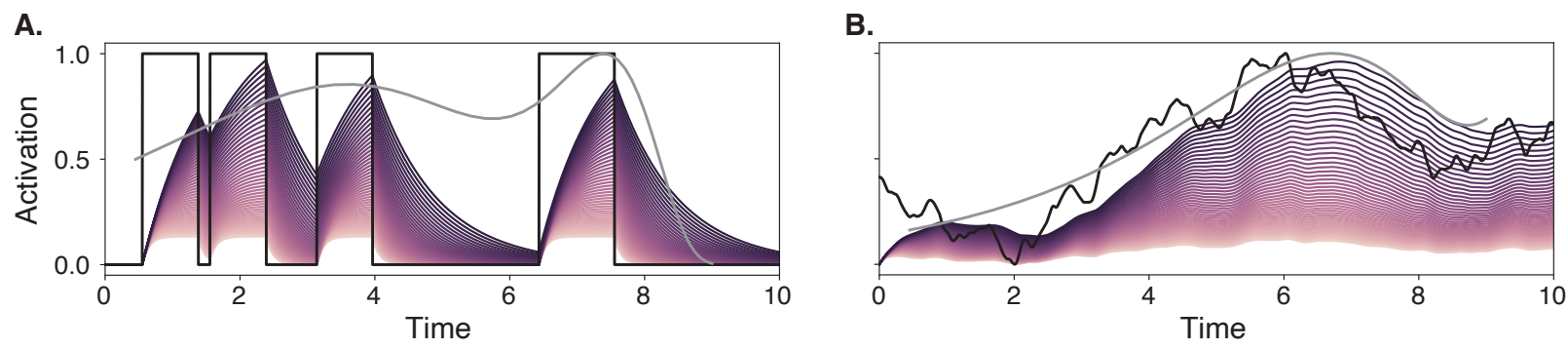

Figure 3: Temporal blurring of ongoing experience in one feature dimension. A. Binary feature. The black line denotes a time course of a single binary feature, $f(t)$. Conceptually, the times when the black line takes on a value of 1 may be thought of as the times when a particular type of (repeated) experience occurred. The colored lines denote temporally blurred time courses $y(t, \rho)$ at 50 log-spaced time constants $(\rho)$. Conceptually, these lines act like a set of neural integrators that blur ongoing experiences at different time scales. The gray line denotes the remembered time course, $\hat{f}(t, \hat{t})$ reconstructed from the set of $y$ s evaluated at $t=10$. Conceptually, the gray line reflects a prediction about how someone might remember when the experiences reflected by the black line occurred. Each family of curves has been rescaled to have a maximum activation of 1 and a minimum activation of 0 . B. Continuous feature. This panel is in the same format as Panel A, but here $f(t)$ is a Real-valued random autocorrelated timeseries.

1. Our ongoing experiences (internally and externally driven thoughts) are integrated and represented at multiple timescales. Thoughts integrated over longer timescales serve as a context for thoughts integrated over shorter timescales.

2. Thoughts that are co-active become associated. Critically, this includes thoughts that are integrated at different timescales. In other words, the evolving contexts in which we interpret information specific to each new moment become an integral part of our memories of each new moment.

3. The current blend of thoughts active in our mind (which will include representations being integrated at a range of different timescales) serves as a retrieval cue, leading us to remember other experiences that were previously associated with a subset of our currently active thoughts.

4. When we remember prior experiences, the contextual thoughts associated with those expe- 
riences are reactivated in our mind. In turn, this leads to a new blend of contextual thoughts, which will become associated, further cue memory, and so on. This implies that remembering an experience entails reactivating thoughts about that experience and other related experiences (that share contextual information). It also implies that each time we remember a prior experience, our memory of that experience will be colored by the contextual thoughts we had at the times of each remembering.

One prediction of this theoretical framework is that recent experiences will be remembered with greater fidelity than experiences from our distant past. For example, this may be seen in Figure 3, whereby the time courses nearest to the end of the interval shown are reconstructed (gray line) with greater temporal fidelity than earlier timepoints, which are temporally blurred together. This is consistent with a substantial literature on temporal judgements that suggests that, equating for ground truth duration, people remember more recently experienced moments as having passed by more quickly than moments from the more distant past (Droit-Volet and Wearden, 2015; Joubert, 1990; Lemlich, 1975; Sahakyan and Smith, 2014; Wearden, 2015). Because, by definition, context representations change gradually (relative to the aspects of our experience being contextualized), the blend of contextual thoughts now will tend to be most similar to the blend of contextual thoughts from other recent moments leading up to now. Our ability to retrieve thoughts about our recent past will be facilitated by their associations with other gradually evolving thoughts that are still active.

In addition to describing how we remember the passage of time along different portions of our autobiographical timeline, this framework also describes how we might mentally revisit our prior experiences. Equation 6 provides a mathematical specification of how the population activity of a set of neural integrators at a given moment can be used to mentally reconstruct the time course of our experiences leading up to that moment. In other words, the equation describes how a single neural pattern (from one moment in time) can reflect the spread of thoughts over an extended time interval. This framework provides one possible explanation of where in time we go 
when we remember: to some extent we revisit every moment along our autobiographical timeline simultaneously. According to this view the concept of a single "moment" in time is meaningful with respect to our subjective experience only insofar as it provides a conceptual reference point for describing the origins (in time) of our current blend of thoughts (as in Fig. 1; each coordinate along the $x$-axis of the figure might be considered to be a single "moment"). Note that, although the example shown in Figure 3 and specified in Equation 6 reflect the experienced and remembered time course of only one feature dimension, this framework may be easily extended by constructing an analogous set of neural integrators for each of several feature dimensions.

Another key prediction of context-based memory models pertains to when we initiate recalls spontaneously in our ongoing experiences. A common process underlying each of these models is that the parallel representations of ongoing experiences active at the same moment become associated. This means that when a current experience is similar to a prior experience (with respect to ongoing representations at any combination of timescales), those prior experiences will be spontaneously remembered. This remembering happens in a graded fashion; the more an ongoing experience matches representations associated with a given prior experience, the more the thoughts associated with that prior experience will be brought to mind. For example, in the airport sequence depicted in Figure 2, thoughts about being hungry might bring to mind other times when you were hungry- and even more strongly, other times when you were feeling hungry at the same airport. This process explains how, when we experience something similar to what we have experienced in the past, we can bring those prior experiences to bear in deciding how to optimize our behaviors and generate expectations about what might happen next (e.g., finding food in the airport).

\section{A neural basis for hierarchical temporal representations}

The notion that our brain maintains parallel mental representations drifting at different time scales is supported by a growing body of neuroscientific evidence. A series of elegant functional magnetic resonance imaging and electrocorticographic studies (Aly et al., 2018; Hasson et al., 2008; Honey 
et al., 2012a; Lerner et al., 2011) defined the concept of a temporal receptive window for each neocortical area, describing the timescale of events to which it is most sensitive. Subsequent work has shown that this hierarchy of drifting mental representations plays a central role in how we remember continuous experiences and segment our experiences into discrete events (Baldassano et al., 2017). This work mirrors the discovery of time cells in the rodent hippocampus that respond preferentially to a specific time relative to a temporal reference (e.g., time elapsed since starting to run on an exercise wheel), whereby each cell appears to integrate incoming information at a different rate (MacDonald et al., 2011; Pastalkova et al., 2008) and the population activity serves to represent information at a range of timescales (Mau et al., 2018). Other work suggests that the lateral entorhinal cortex also plays a role in integrating information across a wide range of timescales (Tsao et al., 2018), supporting precise temporal recall (Montchal et al., 2019). Time cells have been hypothesized to play the role of neural integrators, such as those displayed in Figure 3 (Shankar and Howard, 2012). How hippocampal time cells might be related to neocortical temporal receptive windows remains an important open question.

\section{Testing theories of context reinstatement}

Although there is abundant evidence that contextual information plays an important role in memory, each tennet of context reinstatement theories of memory must also be considered in light of potential alternative theories. A challenge to studying many psychological processes, including context reinstatement, is that psychological processes often have no direct overt manifestation. For example, we are continually engaged in encoding our ongoing experiences and retrieving information about our prior experiences- but those processes can only be observed indirectly when they affect observable behavior or (in the laboratory) neural activity patterns. 


\section{Behavioral evidence for context reinstatement}

Behaviorally, one can study context reinstatement in the laboratory by asking participants to recall their prior experiences (e.g., from before they began the experimental testing session), or by exposing the participant to new experiences or stimuli and then testing their memory. These memory tests can take many forms, including free recall (unconstrained free-form descriptions of an experience or remembered stimuli), serial recall (e.g., asking someone to recall a sequence of events or stimuli in a particular order), recognition memory (e.g., making a judgement about whether a given stimulus has been encountered before), and priming (e.g., studying the effects of having been exposed to one set of experiences or stimuli on memory for, or responses to, another set of experiences or stimuli).

The behavioral effects of context reinstatement may be observed by characterizing which experiences or stimuli are remembered (or forgotten), the order and timing in which the memories are retrieved (or the delays between successive recalls), the quality of the remembered information (e.g., how well it matches the original experience or stimulus), and in false memories (i.e., memories for experiences or stimuli the participant was never exposed to). One behavioral pattern that has been interpreted as evidence for context-based accounts of memory is called the contiguity effect (Healey and Kahana, 2014; Kahana, 1996). This refers to the observation that, given that someone has just recalled a previously encountered event or stimulus, they will tend to next remember another event or stimulus they previously encountered at a nearby time. The contiguity effect is scale invariant, meaning that the same general pattern is observed at short and long timescales. For example, remembering an event from a particular day might bring to mind other events from the same day. Similarly, remembering a given year of your life might bring to mind other years when you were a similar age. Contiguity effects across a range of timescales may be explained naturally by context reinstatement models. Two properties of these models combine to explain contiguity. First, slowly drifting contextual representations become associated with temporally adjacent experiences or stimuli. This means that experiences that occur nearby in time 
will tend to be associated with similar contexts. Second, the contextual features associated with a memory are reinstated during recall, which then cues recall for other experiences associated with similar contexts (which, by the first property, will tend be temporally nearby). According to this view, contiguity is timescale invariant because closer-in-time experiences will tend (all else being equal) to be associated with relatively more similar contexts than further-in-time experiences, regardless of how distant in time those experiences are in absolute terms. As we will see later in this chapter, the ways we organize and catalogue our memories with respect to the timing of our experiences are mirrored in how we organize our memories with respect to non-temporal information, such as conceptual or semantic content, spatial information, etc.

The fact that context reinstatement models can explain the timescale invariant nature of contiguity does not necessarily mean that these models are the only possible explanation for this phenomenon. Another broad class of models that can explain some aspects of contiguity are termed dual store models (e.g., Atkinson and Shiffrin, 1968). These models posit the existence of separate short-term and long-term memory storage mechanisms. According to these models, each new experience (e.g., a percept from an ongoing experience, an item on a studied list, an idea from an ongoing conversation, etc.) occupies a position in a limited capacity short-term (working) memory buffer. The contents of this buffer may be conceptualized as the "current mental state" (i.e., the collection of thoughts someone is having at a particular moment). The contents of this buffer become associated in long-term memory, which has effectively limitless capacity. Since temporally proximal experiences will tend to occupy the short-term memory buffer at the same time, these models provide an alternative explanation for contiguity. However, these models cannot explain direct temporal associations at timescales beyond the capacity of the short-term memory buffer. Therefore dual store models do not account for the timescale invariant nature of contiguity. 


\section{Neural evidence for context reinstatement}

Neuroimaging studies in the laboratory enable researchers to measure brain activity patterns as experimental participants learn and recall information. One can then examine brain patterns at the time of encoding (i.e., the time when the original experience or stimulus is encountered) and retrieval (i.e., the time when the experience or stimulus is recalled). An example of this type of analysis is displayed in Figure 4A. Manning et al. (2011) recorded from electrodes implanted in the brains of neurosurgical patients undergoing a treatment for drug-resistent epilepsy. The patients studied and verbally recalled a series of random word lists; the researchers compared the neural activity patterns recorded just before the patients vocalized each recall to the activity patterns recorded while the patients studied the about-to-be-recalled word on the list, and other nearby words on the list. The neural similarity curves in Figure 4A show that the brain patterns just prior to vocalizing a recall were similar to the patterns recorded as the patients were studying the aboutto-be-recalled word, but also (to a lesser extent) to the activity patterns recorded when the patient studied other words that were nearby on the list. This result is consistent with the account of context reinstatement models: it suggests that the patients' brains maintained a gradually evolving neural representation that was reactivated just prior to remembering each studied word. Further, just as context reinstatement models predict that contiguity (Fig. 4B) arises due to context reinstatement, the patients who showed the strongest neural signatures of context reinstatement also showed the strongest tendency to successively recall nearby list items (Fig. 4C). Similar neural signatures of context reinstatement have also been observed in neurosurgical patients during recognition memory tasks (Folkerts et al., 2018; Howard et al., 2012) and paired-associates learning (Yaffe et al., 2014).

Although these neural similarity findings are consistent with context reinstatement, they do not (by themselves) directly rule out alternative accounts, such as dual store models. For example, the neural signature of temporal context reinstatement shown in Figure 4A could also be explained by a short-term memory buffer that was gradually updated during study as new words are encountered, 
A.

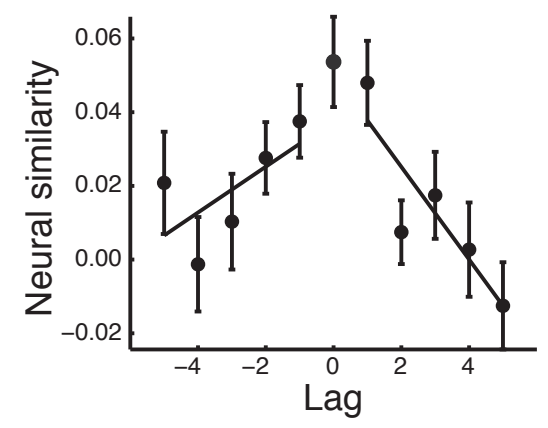

B.

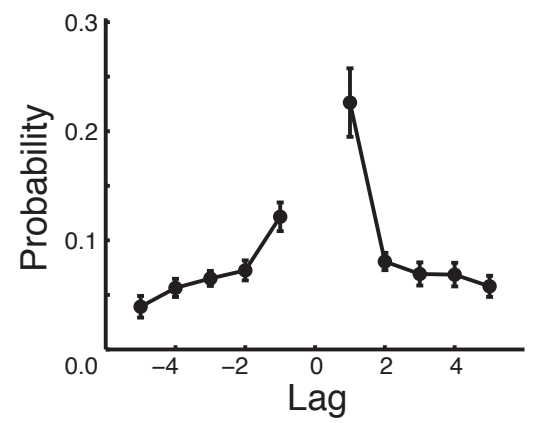

C.

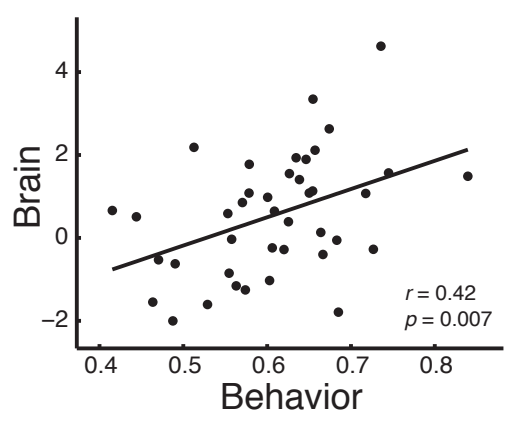

Figure 4: Neural correlates of temporal context reinstatement during random word list free recall. A. Neural activity patterns recorded just prior to recall of each word on a studied list are compared to the patterns recorded during the encoding of the same word (lag $=0$ ) and surrounding words. Here lag refers to the positional difference on the studied list between the recalled word and other nearby words on the list. B. Conditioned on having correctly recalled a given word, the panel displays the probability of next recalling other words on the list, as a function of their relative study positions (lags) to the just-recalled word. C. Each dot reflects data from a single experimental participant. The $x$-coordinates denote the strength of the behavioral contiguity effect (i.e., the steepness of the conditional response probability curves in Panel B), and the $y$-coordinates denote the strength of the neural contiguity effect (i.e., the steepness of the neural similarity function in Panel A). This figure is adapted from Manning et al. (2011).

and where words that occupied the buffer concurrently were associated. Taken together, these studies show that the neural patterns exhibited while remembering a list item are similar to the neural patterns exhibited during study of a range of list items.

The above studies sought to identify neural correlates of context reinstatement by isolating gradually evolving neural patterns and comparing patterns recorded at different timepoints. An alternative approach to studying the neural correlates of context is to leverage advances in neural decoding. This family of approaches attempts to map neural activity patterns onto stimulus features or category labels (Haxby et al., 2001; Huth et al., 2016, 2012; Mitchell et al., 2008; Norman et al., 2006; Shinkareva et al., 2008). After training a neural decoder (e.g., on a subset of the data), one can design memory experiments where to-be-memorized stimuli are specially selected to be distinguishable by the decoder(s). For example, items on a studied list might be drawn from a 
small number of discrete and easily differentiable categories.

In addition to using neural decoders to track brain activity related to the remembered items themselves (Polyn et al., 2005), one can present these traceable stimuli incidentally (e.g., interspersed between the to-be-remembered list items) to study their effects on context. For example, Gershman et al. (2013) designed a neuroimaging list learning paradigm whereby participants memorized two random lists of object images (car, ball, apple, watch, etc.) over two study sessions (separated by 48 hours). During the first study session, the participant repeatedly viewed object images from the first list. Between each successive object image, the participant passively viewed a series of images of outdoor scenes (mountains, forests, etc.). The researchers hoped these scene images would become integrated into the drifting context representations associated with the list. In a second study session, participants studied a new list of object images. Again, the list was repeated several times, but no scene images were shown between the list item presentations. Finally, in a third session (48 hours later), the participants were tested on their source memory- their ability to remember which list each object image came from (i.e., the first or the second list). When participants mistakenly attributed second list items to the first list, their brains exhibited scene-related neural patterns (indicating that they were bringing the scene images from the first list to mind). In this way, the study provided neural evidence for a context reinstatement account of source memory errors. In a related study, Manning et al. (2016) designed a similar neuroimaging list-learning paradigm. On each of several trials of a list method directed forgetting experiment, they had participants study a sequence of two random word lists. Between the presentations of words on the first list, the researchers flashed a series of scene images on the screen. Next, participants were told to either "remember" or "forget" the words from the first list, prior to studying the second list (which did not contain scene images). Analogous to the study by Gershman et al. (2013), the researchers hoped that these scene images would become integrated into the context associated with the first list. The researchers found that when people were told to forget the first list, scene-related brain activity patterns were substantially reduced. This suggests that we can intentionally forget 
by flushing the associated contextual features out of our active mental state (Sahakyan et al., 2013; Sahakyan and Kelley, 2002).

\section{Confounds of autocorrelation and semantic similarity}

We can infer the role that context reinstatement plays in memory through contextual effects on behavior (e.g., facilitating recall of context-matched experiences and stimuli) and neural activity patterns (e.g., neural similarity between experiences or stimuli associated with similar contexts). However, how can we know that these phenomena are truly contextually mediated?

To explore the bounds of what we can and cannot infer about context reinstatement, let us try a thought experiment. Reflect on an experience you have nearly every day, such as walking to class, commuting to work, going to the gym, etc. Consider how you might mentally focus in on one specific moment of one specific day's variant of your chosen daily experience (e.g., a bird that flew in front of you on the way to class). In the lab, how might we go about conceptually distinguishing that particular moment from other moments within the same day's experience, or from other days' experiences, or other similar moments (e.g., bird-related) from other experiences? There are several substantial challenges that must be addressed. Because our ongoing experiences and thoughts tend to be autocorrelated, any given moment will tend to exhibit similar properties (and evoke similar thoughts) to other temporally nearby moments. Our ability to pin down the "one specific moment" an experimental participant is reflecting on will be limited by those autocorrelations. Further, repeated experiences (e.g., walking to class each day) may themselves appear similar (e.g., similar landmarks encountered, similar rates of visual flow, similar people encountered along the way, similar starting and ending destinations, etc.). These similarities can have behavioral and neural ramifications. For example, frequently repeated experiences may be schematized such that the unique contextual attributes are lost in favor of the aspects of the experience that are common and predictable across repetitions (Ranganath and Ritchey, 2012). Schema representations can have similar behavioral and neural manifestations, even when the specific details differ (Baldassano 
et al., 2018). In general, any experiences or stimuli that share semantic or conceptual attributes may appear similar (behaviorally and neurally), whether or not those memories are directly associated. Examining which memories we are likely to transition to next (e.g., a primary behavioral manifestation of context reinstatement) or which prior neural activity patterns our brain currently matches (e.g., a primary neural manifestation of context reinstatement) often fail to differentiate between specific exemplars of a given experience or stimulus.

Beyond posing a methodological challenge to studying context reinstatement, similarity confounds can also have deep psychological implications. For example, when we revisit a past experience, the above studies suggest that we mentally spread our thoughts over a range of timepoints, as in Figure 1. But can we detangle this "mixture" view from other possible accounts? For example, suppose instead that when we remembered, we mentally revisited only one specific moment- the time at which we experienced what we are remembering (this is termed episodic sampling). If that specific moment happened to share features with other moments from our past, would this look fundamentally different from a mixture model, from either a behavioral or neural perspective?

One way of identifying instances of episodic sampling is to tag specific episodes with contextual markers that are traceable using neuroimaging (Bornstein and Norman, 2017), analogous to methods used in several of the neuroimaging list-learning studies mentioned above (Gershman et al., 2013; Manning et al., 2016). One may then use the neural activity patterns associated with the contextual tag to estimate the degree to which the contextual features associated with a specific episode have been brought to mind. Bornstein and Norman (2017) showed that when participants remembered a specific prior experience (episode), their subsequent decisions were influenced not only by other episodes that occurred nearby in time, but also by other episodes that occurred in similar contexts. This is consistent with the notion that we make decisions by integrating across (potentially many) distinct prior experiences that we deem relevant (for review see Shohamy and Daw, 2015).

Another means of teasing out potential confounds due to autocorrelations or semantic relat- 
A. Video trajectory

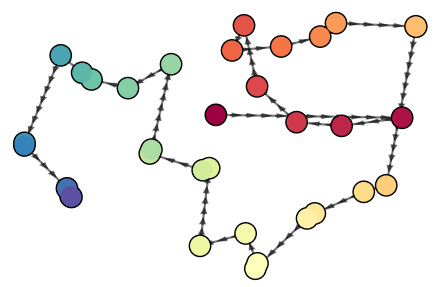

B. Recall trajectory

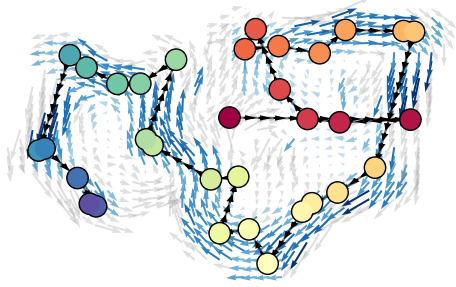

Figure 5: How do experiences, and our memories of those experiences, unfold over time? A. The trajectory through topic space taken by a television episode. The topic trajectory taken by a 50 minute television episode, projected onto two dimensions. Each dot indicates an event identified using a Hidden Markov Model; the dot colors denote the order of the events (early events are in red; later events are in blue), and the connecting lines indicate the transitions between successive events. B. The trajectory through topic space taken by participants' recalls of the television episode. The average two-dimensional trajectory captured by 17 participants' recalls of the episode from Panel A, with the same format and coloring as the trajectory in Panel A. The arrows reflect the directions of the average transitions taken by participants whose trajectories crossed that part of topic space; blue denotes reliable agreement across participants. This figure is adapted from Heusser et al. (2018a).

edness in the remembered stimuli is to build explicit models of how the to-be-remembered information varies over time. One example of this approach comes from a recent study by Heusser et al. (2018a). The researchers analyzed data, collected by Chen et al. (2017), from participants who watched an episode of the BBC television show Sherlock and then verbally recounted what happened in the episode. The authors used topic models (Blei et al., 2003) applied to humangenerated annotations of each camera shot in the episode to characterize the episode's content with a timeseries of high-dimensional semantic feature vectors. These topic vectors describe the mix of "themes" present in each moment of the episode. The sequence of topic vectors over the course of the episode traces out a topic trajectory through a high-dimensional feature space (Fig. 5A). The sequence of topic vectors for a given participant's recalls traces out an analogous trajectory (Fig. 5B). This characterization provides a geometric framework for comparing the shapes that reflect how naturalistic experiences unfold over time, to the shapes of how we remember those experiences later. One can then use the geometric transformations needed to map an experience's trajectory onto the trajectory of its later remembering to characterize the quality and content of 
memory.

When participants in list-learning studies display evidence of leveraging semantic information in organizing their memories (e.g., Manning and Kahana, 2012; Manning et al., 2012; Wixted and Rohrer, 1994), it is difficult to explicitly test whether this specifically reflects the integration of information across the experiences of studying the different list items. However, it is certainly the case that integrating information across different list items is not required to memorize the lists. By contrast, in more "naturalistic" stimuli like the one used by Chen et al. (2017), forming those across-event associations is critical to understanding the underlying narrative. Building explicit models of the stimulus dynamics, and of how the stimulus is remembered later, enables researchers to partially separate out the structure of the stimulus from the structure of people's memory for the stimulus. The way our memory systems distort our experiences when we remember them later can tell us which aspects of our experiences are leveraged in building the scaffoldings of our memories.

\section{Non-temporal contextual feature spaces}

The relative timing with which our ongoing experiences and thoughts fade in and out of our mental states is the dominant property people use to organize their episodic memories (Healey and Kahana, 2014). However, our memory systems also leverage other non-temporal dimensions in organizing our memories. Many of the general principles that govern how we represent temporal information also apply in analogous ways to how we represent non-temporal dimensions such as space, semantics (meaning), situations, and emotions. There is evidence, for at least several of these domains, that the corresponding mental representations are organized hierarchically in a scale invariant manner, and that the underlying representations exhibit autocorrelations and boundaries that are analogous to scale invariant temporal hierarchies, autocorrelations, and event boundaries. Most theories of context reinstatement follow a computational approach, taken by Polyn et al. (2009) in developing their context maintenance and retrieval model, whereby stimuli and un- 
A.

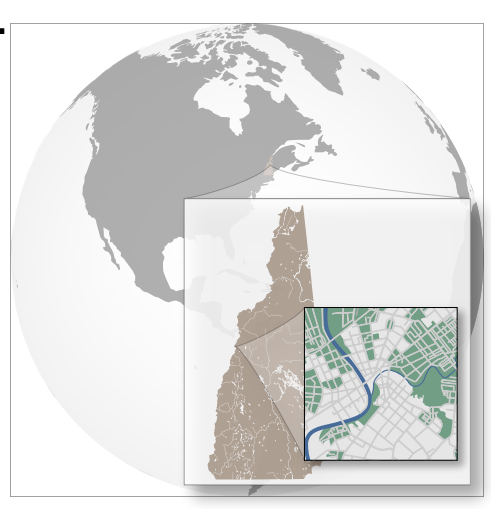

B.

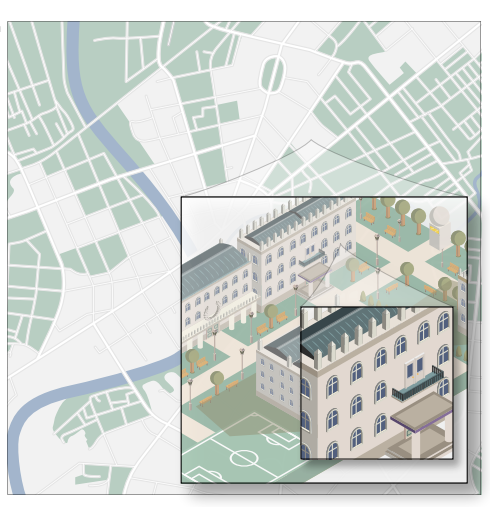

C.

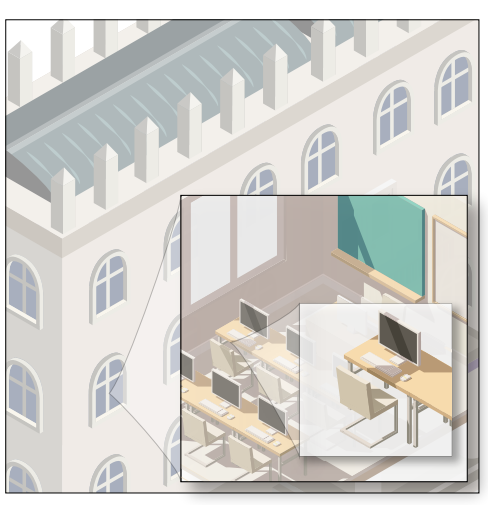

Figure 6: Spatial hierarchies. A. Macro scale. A city's location is conceptualized within a state, whose location in turn is conceptualized within a country. B. Meso scale. A building's location is conceptualized within an associated campus, whose location in turn is conceptualized within a city. C. Micro scale. A desk's location is conceptualized within a room, whose location in turn is conceptualized within a building. Each spatial concept (room, building, campus, city, state, country) provides a spatial context for relatively more micro-scale concepts, and is in turn contextualized by more macro-scale concepts.

folding experiences are represented as high-dimensional feature vectors that reflect (in different dimensions) the temporal, semantic, conceptual, spatial, situational, and task-related properties of each memory. This allows one to characterize how contextual features along these different dimensions affect memory.

\section{Spatial context}

Just as events that we experience nearby in time are often conceptually related, nearby spatial locations often also share conceptual attributes. For example, the components of a single object tend to occupy nearby spatial locations, and nearby objects often share conceptual or functional properties.

Broadly, there are two categories of spatial information to which our memory systems are sensitive. First, within a single visual scene (e.g., a photograph or our visual perspective from a single viewpoint), we leverage the relative positions and spatial relations between different scene elements in remembering what we see and in integrating across memories of different scenes (Robin 
and Olsen, 2019; Tsitsiklis et al., 2019). Second, within our broader environment, our own spatial location at the time we experience a stimulus or event becomes part of that memory's context and affects how we organize and relate our corresponding memories of those stimuli or events (Miller et al., 2013a), and of the spatial layout of the environment (Manning et al., 2014). Our memory systems leverage these spatial properties of our environments-and our locations within our environments, at a wide range of spatial scales-to organize our memories (Fig. 6).

The neural basis of our cognitive map-i.e., the neural machinery that enables us to keep track of our spatial position within an environment-is relatively well understood. The hippocampus contains specialized place cells that modulate their firing rates according to our current location (Ekstrom et al., 2003; O'Keefe and Dostrovsky, 1971; Tolman, 1948). In turn, these place cells are informed by grid cells in the entorhinal cortex that modulate their firing rates when one's current location is at any vertex of a triangular lattice that tiles the environment (Jacobs et al., 2013; Moser et al., 2008). Modeling work has described how populations of grid cells with vertices tiling an environment at different spatial scales can explain the formation of place cells' receptive fields (Fuhs and Touretzky, 2006; McNaughton et al., 2006; O'Keefe and Burgess, 2005; Solstad et al., 2006). Finally, postsubicular head direction cells respond when the head is oriented along a particular direction (Sargolini et al., 2006; Taube et al., 1990), and subicular border cells respond at the boundaries or obstacles to navigation within an environment (Savelli et al., 2008; Solstad et al., 2008). Taken together, these populations of cells provide sufficient information to localize and orient an individual within the surrounding environment. When we remember, the cells that represented our spatial position at the time of our original experience increase their firing rates (Miller et al., 2013b; Moser et al., 2015). Further, when the properties of our spatial environment change abruptly (such as when we exit a room and enter another), our ability to retrieve information from before the change is impaired, controlling for the amount of time elapsed (Radvansky and Copeland, 2006). This work provides additional evidence that some part of the mental context we use to probe our memories is related to spatial location and also suggests that spa- 
tial boundaries may be leveraged in a similar way to temporal event boundaries with respect to differentiating and organizing our memories (Brunec et al., 2018; McKenzie and Buzsáki, 2016).

Analogous to how time-responsive cells in the hippocampus and cortex exhibit preferences at different temporal scales, grid cells (Fyhn et al., 2004; Hafting et al., 2005) and place cells (Kjelstrup et al., 2008; Wilson and McNaughton, 1993) also exhibit receptive fields at different spatial scales. In other words, our spatial representations are to some extent scale invariant. Although both time- and place-responsive neurons may be localized to cell populations within the hippocampus, the potential functional associations between time cells and navigationally relevant cells (including place cells) remains an open question of considerable interest (Collin et al., 2015; Howard and Eichenbaum, 2013, 2014).

\section{Semantic context}

Physically, we experience the world as a constant stream of information. Yet we are able to make conceptual sense of our physical and psychological surroundings, parsing aspects of our experience into manageable (and often discrete) functional and conceptual units that we can leverage in considering, remembering, and communicating our external and internal experiences. Semantic models attempt to quantify the meanings we ascribe to specific words, and the conceptual relations and associations between different words.

Researchers have taken two general approaches to modeling semantic information. The first approach, broadly termed word embedding models, is to derive computational algorithms for analyzing large text corpora to automatically determine the meanings of words based on how they are used. For example, latent semantic analysis (Landauer and Dumais, 1997) uses word cooccurrence statistics (i.e., how often pairs of words occur in the same documents contained in the corpus) to derive a unique feature vector for each word. The feature vectors are constructed so that words that co-occur more frequently have feature vectors that are closer (in Euclidean distance). Related approaches, such as latent dirichlet allocation (Blei and Lafferty, 2006; Blei et al., 2003) 


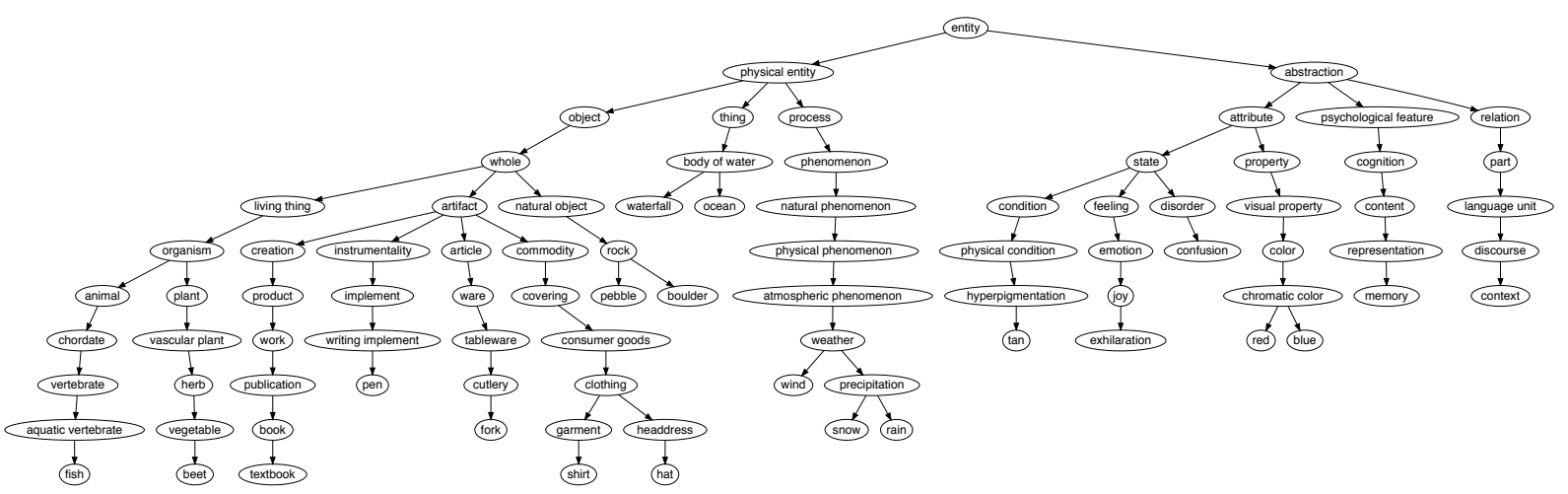

Figure 7: Semantic network. Each node is an instance of its parent. Nodes that share lower-level parents also share lower-level conceptual features. This tree diagram displays a subset of Princeton WordNet (Miller, 1995). Compare this parallel distributed processing approach to visualizing semantic relations to the word embeddings of events in a television episode displayed in Figure 5.

attempt to explicitly model the underlying causes of word co-occurrences by automatically identifying the set of themes or topics reflected across the documents in the corpus. More recent work on these types of semantic models, including word2vec (Mikolov et al., 2013) and the universal sentence encoder (Cer et al., 2018), use deep neural networks to attempt to identify the deeper conceptual representations underlying each word. An example of how this general approach may be applied to memory data is shown in Figure 5.

An alternative approach falls under a conceptual framework called parallel distributed processing (McClelland et al., 1986; Rumelhart et al., 1986; Seidenberg and McClelland, 1989). Whereas word embedding approaches generally attempt to capture the relative similarities between words using feature vectors with arbitrary feature dimensions (i.e., typically not directly human interpretable), parallel distributed processing approaches define an explicit hierarchy of semantic features. Figure 7 displays a semantic network derived from Princeton WordNet (Miller, 1995) for several example words. For any two nodes of the semantic network, one may use graph theoretic measures, such as the number of edges in the shortest connecting path between the nodes (Dijkstra, 1959) to estimate the semantic similarities between the nodes' corresponding concepts. Note that, although they are not typically applied together, parallel distributed processing approaches are not 
necessarily mutually exclusive with word embedding approaches. For example, within a semantic network, each node may be assigned a separate feature dimension. To generate a feature vector for a given concept, one may then set the corresponding feature dimension to 1 for that concept's node, and for any dimensions that correspond to ancestors of that node (with all other dimensions set to 0). This will result in binary feature vectors that are more similar (closer in Euclidean distance) for nodes that share a larger number of common ancestors. In turn, these binary feature vectors may be embedded in lower-dimensional spaces to facilitate visualization (Heusser et al., 2018b). The parallel distributed processing approach to modeling semantic relations explicitly captures the scale invariant nature of meaning. For example, as shown in Figure 7, the semantic network captures the notion that different types of aquatic vertebrates (e.g., fishes, eels) are more similar to each other than an aquatic vertebrate is to a plant (e.g., beets). In turn, these different types of organisms are more similar to each other than any given organism is to a weather pattern (e.g., snow). Effectively, each parent node in the tree provides a semantic context for its children.

A number of studies indicate that participants spontaneously form associations between semantically related words on random word lists, such that subjects recall semantically related words consecutively even when the presentations of those related words are separated in time (e.g., Manning and Kahana, 2012; Manning et al., 2012; Wixted and Rohrer, 1994). This suggests that our memory systems pick up on statistical regularities, even in ostensibly "random" stimulus sequences, and that we leverage those regularities when we recall our past (Polyn et al., 2009). Other work suggests that our memory systems may also leverage these regularities to parse our ongoing experiences into discrete events (Schapiro et al., 2013; Shapiro, 2019). Conceptual boundaries (e.g., distinctions between semantic categories; Brunec et al., 2018) can also shape how we experience and remember conceptual information.

Neuroimaging studies indicate that our brains represent semantic information via patterns of activity that are distributed across the neocortex (Huth et al., 2016, 2012; Mitchell et al., 2008; Shinkareva et al., 2008). The most detailed studies to date were carried out by Huth et al. (2012) 
and Huth et al. (2016). Huth et al. (2012) had participants watch movies as they underwent functional magnetic resonance imaging. Human annotators labeled objects and actions in each frame of the movies that were also in the WordNet lexicon. This yielded a time course for each object and action reflecting when the corresponding term was present in each moment of the movies. The researchers examined how the timeseries of each voxel (a tiny volumetric unit reflecting neural activity in one small part of the brain) related to the timeseries of each term. The researchers found that the voxels' preferences (i.e., the terms to which they responded most readily) were spatially distributed across the neocortex in an organization that roughly corresponded to the high-order categories contained in WordNet (Fig. 7). For example, voxels that preferentially responded to tool-related terms tended to be grouped together, and tended to be closer together on the cortical surface to other equipment-related terms than to less semantically similar terms such as plantrelated or animal-related terms. Huth et al. (2016) used an analogous approach to study voxel responses as participants listened to auditory stories. They found that the semantic maps they discovered were similar across individuals, suggesting that at least some aspects of the meanings we ascribe to concepts are represented similarly across different people's brains. Related work suggests that these maps may even be conserved across native speakers of different languages and across cultures (Honey et al., 2012b).

\section{Situational context}

Although most models of semantic meaning make the simplifying assumption that our internal semantic representations are static (although see Blei and Lafferty, 2006), the current situation we find ourselves in, and the set of goals we are currently trying to achieve, can affect how we consider the information content of our ongoing experiences. The posterior medial system is a densely interconnected set of brain structures that plays a critical role in representing situational contexts (Ranganath and Ritchey, 2012). This system includes the parahippocampal cortex, retrosplenial cortex, the mammillary bodies, anterior thalamic nuclei, presubiculum, parasubiculum, 
posterior cingulate, precuneus, angular gyrus, and ventromedial prefrontal cortex. Ranganath and Ritchey (2012) suggest that these regions collectively match incoming information about ongoing experiences to internal situation models that define a set of expectations about specific situations. For example, the situation model for eating at a restaurant might describe the general properties of restaurants, the typical sequence of events during a restaurant outing, restaurant etiquette, types of food expected to be on the menu, expected prices of different menu items, expected wait times, expected behaviors of other people in the restaurant, how enjoyable different aspects of the experience are expected to be, etc. (Zacks et al., 2007; Zwaan and Radvansky, 1998).

Situation models may also be organized hierarchically. For example, situation models that involve interactions with other people may each be associated with a particular broad set of behaviors and expectations. Restaurant-related situation models may be associated with a subset of those behaviors and expectations. And a situation model related to a specific type of restaurant (e.g., family-style Chinese restaurants, sandwich shops, Michelin starred restaurants, etc.) might each be associated with a still more focused subset of restaurant-related behaviors and expectations. Goals may also modulate situation models. For example, on a visit to a restaurant on a first date one may behave differently, and have different goals and expectations, than during a lunch meeting with colleagues, a family dinner, or a dinner with close friends- even if all of these scenarios take place in restaurants.

When ongoing experiences stop being well-explained by the current situation model, we infer that an event boundary has occurred. Theories of context and memory predict that the act of flushing out the previous situation model from one's current mental state, and loading in a new one relevant to the next perceived situation, will cause mental context to change abruptly (Reynolds et al., 2007; Shankar et al., 2009). In turn, this will impair memory for experiences that occurred prior to the event boundary (Ezzyat and Davachi, 2011; Radvansky and Copeland, 2006; Swallow et al., 2011, 2009). This may serve to focus memory search on recent experiences within the current situation. 
Computational models that posit that contextual representations drift gradually as we integrate incoming information (e.g., Figs. 2, 3) do not typically incorporate computational machinery that could explain how the current situation model might be changed or updated at event boundaries. One extension of these models, termed the predictive temporal context model (Shankar et al., 2009), updates the current state of context with a prediction of what will happen in the next moment (rather than solely integrating ongoing experience). When these predictions fail to accurately account for the observable future, this could provide a mechanism for triggering an event boundary whereby the current state of context is "reset" and a new situation model is incorporated into the new active state of mental context. Subsequent work (e.g., Gershman et al., 2012) has also shown how temporal context models are related to other predictive models such as the successor representation (Dayan, 1993). The successor representation is a model of how predictions of future states (successors) may be made in a generalized way across similar or related states (also see Momennejad et al., 2017).

\section{Emotional context}

Part of our subjective ongoing experiences reflects what is physically happening to us, as measured by our senses (i.e., what we see, hear, touch, taste, and smell). As described above, we can also incorporate conceptual and situational information into our mental context, shaping how we process, remember, and form predictions about our experiences. How do these principles apply to internally generated aspects of our subjective experiences, such as our emotions?

From a computational perspective, emotional thoughts need not be any different than externally driven thoughts. Indeed, a growing body of work suggests that memory encoding and retrieval is influenced by the emotional content of remembered information (Bower et al., 1981, 1978; Long et al., 2015; Talmi et al., 2019). Emotions may be represented using the same word embedding models as other semantic concepts. However, a substantial challenge to studying how emotions affect context and memory is that there is considerable variability across people with respect to 
which specific emotions are triggered by a given experience, how a given emotion is experienced, and which emotions are able to be experienced (Barrett, 2006). Whereas the physical aspects of an experience may be tightly controlled in a laboratory setting (e.g., the timeseries of pixel intensities and auditory waveforms presented to an experimental participant), controlling a participant's response to a given physical stimulus is less straightforward. Although there is evidence that the emotions we experience are directly tied to underlying physiological processes such as neurotransmitter release (Barrett et al., 2007), our subjective experiences of those underlying physiological processes vary across people, as do the times at which those processes occur in different people even in response to a consistent (physical) stimulus. For example, the ways we interpret and respond to our external world emotionally are driven in part by our idiosyncratic prior experiences, goals, and expectations (Roy et al., 2012).

One advance to studying idiosyncratic emotional states comes from a recent study by Chang et al. (2018). They had participants watch an episode from the character-driven dramatic television show Friday Night Lights while undergoing functional magnetic resonance imaging. They focused their analyses on activity patterns in the ventromedial prefrontal cortex, a brain region hypothesized to represent emotional information. Whereas primary sensory regions (e.g., primary visual cortex) exhibited highly stereotyped response patterns across individuals who watched the episode, the ventromedial prefrontal cortex responses were different across people, with respect to their temporal and spatial patterns. The researchers applied hidden Markov models to the neuroimaging data to automatically identify state transitions- moments when the neural response patterns changed particularly abruptly (i.e., transitions) between extended periods of relative stability (i.e., states). Although the times in the episode that these state transitions occurred varied across people, the activity patterns in several of the states were similar across people. This suggests that there are some activity states, represented in the ventromedial prefrontal cortex, that are similar across people (even if the times at which people enter those states differ across people). In followup experiments, the researchers recorded participants' facial expressions during the episode, 
and had participants rate the moment-by-moment emotional content of the episode. The facial expression and emotional content ratings exhibited discretizeable states that could be identified using hidden Markov models. Like the ventromedial prefrontal cortex responses, the timings of the facial expression and emotion rating state transitions varied across people. Despite variability across people, during several highly emotional moments of the episode, nearly every participant exhibited a simultaneous state change in their ventromedial prefrontal cortex response pattern, facial expression, or emotional ratings. By examining the facial expressions and emotional ratings exhibited during these moments, the researchers identified two states that were common across people. The first state was primarily associated with positive feelings like joy, pride, hope, satisfaction, elation, and relief; the second state was primarily associated with negative feelings like sadness, guilt, and envy. This work implicates the ventromedial prefrontal cortex in ascribing emotional valence to our ongoing experiences (also see Ashar et al., 2017; Kelley et al., 2002; Roy et al., 2012).

\section{Summary and concluding remarks}

Context refers to the blend of internally and externally driven thoughts that we carry into each new moment. The properties of this blend of contextual thoughts can take on different meanings in different domains, but a unifying principle is that higher-level conceptual thoughts provide a context for lower-level conceptual thoughts. These contextual thoughts may be temporal (slowly drifting thoughts contextualize faster drifting thoughts), spatial (larger spaces contextualize smaller spaces), semantic (broader concepts contextualize more specific concepts), situational (high-level situation models contextualize more specific lower-level situation models), and/or emotional (slower drifting and higher order emotional states contextualize faster drifting and more specific emotional states).

Context reinstatement (i.e., reactivating remembered contexts) enables us to associate experiences, environments, concepts, situations, and emotions that share high-level attributes. In turn, 
this enables us to bring our prior experiences to bear on our interpretations of what is happening now. It also enables us to generate predictions about what is likely to happen in the future, given what has happened under similar or related circumstances in the past.

An emerging theme in the study of context reinstatement is an increasing reliance on naturalistic stimuli (e.g., movies, stories, and real-world experiences) that allow researchers to capture neural responses to stimuli that exhibit rich temporal, spatial, semantic, situational, and emotional structure at a range of scales. Because most of our theoretical understanding of context reinstatement has been derived from much more simplistic and tightly controlled experimental paradigms (e.g., list-learning experiments), open questions remain about the degree to which the principles of contextual drift and reinstatement that apply in the domain of list-learning experiments also apply in analogous ways to more naturalistic settings. For example, further study is needed to understand how contextual representations evolve at boundaries (e.g., event boundaries, spatial boundaries, semantic category boundaries, etc.), how the representations of boundaries arise, and how we parse continuous real-world experiences into discrete, memorable, functional units that may be leveraged in optimizing future behaviors and expectations. Several fundamental questions about the neural basis of context reinstatement also remain largely unanswered. For example, while some aspects of the neural representations of temporal, spatial, semantic, situational, and emotional contexts are understood, it is not yet known how these representations might relate or interact.

The ways we revisit the contextual thoughts associated with our past experiences is a central component of the human experience. Our memories of our prior experiences shape how we think of ourselves and others, and how we decide how to behave accordingly. Context reinstatement is a gatekeeper to our memories, determining which aspects of our past we have access to, and which are shut off from our current mental reach. 


\section{References}

Aly, M., Chen, J., Turk-Browne, N. B., and Hasson, U. (2018). Learning naturalistic temporal structure in the posterior medial network. Journal of Cognitive Neuroscience, 30(9):1345-1365.

Ashar, Y. K., Chang, L. J., and Wager, T. D. (2017). Brain and psychological mechanisms of the placebo effect: an affective appraisal account. Annual Review of Clinical Psychology, 13:73-98.

Atkinson, R. C. and Shiffrin, R. M. (1968). Human memory: A proposed system and its control processes. In Spence, K. W. and Spence, J. T., editors, The psychology of learning and motivation, volume 2, pages 89-105. Academic Press, New York.

Baldassano, C., Chen, J., Zadbood, A., Pillow, J. W., Hasson, U., and Norman, K. A. (2017). Discovering event structure in continuous narrative perception and memory. Neuron, 95(3):709721.

Baldassano, C., Hasson, U., and Norman, K. A. (2018). Representation of real-world event schemas during narrative perception. Journal of Neuroscience, 38(45):9689-9699.

Barrett, L. F. (2006). Are emotions natural kinds? Perspectives on Psychological Science, 1(1):2858.

Barrett, L. F., Mesquita, B., Ochsner, K. N., and Gross, J. J. (2007). The experience of emotion. Annual Review of Psychology, 58:373-403.

Blei, D. M. and Lafferty, J. D. (2006). Dynamic topic models. In Proceedings of the 23rd International Conference on Machine Learning, ICML '06, pages 113-120, New York, NY, US. ACM.

Blei, D. M., Ng, A. Y., and Jordan, M. I. (2003). Latent dirichlet allocation. Journal of Machine Learning Research, 3:993 - 1022. 
Bornstein, A. M. and Norman, K. A. (2017). Reinstated episodic context guides sampling-based decisions for reward. Nature Neuroscience, 20(7):997-1003.

Bower, G. H., Gilligan, S. H., and Monteiro, K. P. (1981). Selectivity of learning caused by affective states. Journal of Experimental Psychology: General, 110(4):451-473.

Bower, G. H., Monteiro, K. P., and Gilligan, S. G. (1978). Emotional mood as a context for learning and recall. Journal of Learning and Verbal Behavior, 17:573-585.

Brunec, I. K., Moscovitch, M. M., and Barense, M. D. (2018). Boundaries shape cognitive representations of spaces and events. Trends in Cognitive Sciences, 22(7):637-650.

Buzsáki, G. (2006). Rhythms of the Brain. Oxford University Press, New York.

Cer, D., Yang, Y., Kong, S. Y., Hua, N., Limtiaco, N., John, R. S., Constant, N., GuajardoCespedes, M., Yuan, S., Tar, C., Sung, Y.-H., Strope, B., and Kurzweil, R. (2018). Universal sentence encoder. arXiv, 1803.11175.

Chang, L. J., Jolly, E., Cheong, J. H., Rapuano, K., Greenstein, N., Chen, P.-H., and Manning, J. R. (2018). Endogenous variation in ventromedial prefrontal cortex state dynamics during naturalistic viewing reflects affective experience. bioRxiv, doi.org/10.1101/487892.

Chen, J., Leong, Y. C., Honey, C. J., Yong, C. H., Norman, K. A., and Hasson, U. (2017). Shared memories reveal shared structure in neural activity across individuals. Nature Neuroscience, 20(1):115.

Collin, S. H. P., Milivojevic, B., and Doeller, C. F. (2015). Memory hierarchies map onto the hippocampal long axis in humans. Nature Neuroscience, 18(11):1562-1564.

Dayan, P. (1993). Improving generalization for temporal difference learning: the successor representation. Neural Computation, 5(4):613-624. 
Diana, R. A., Yonelinas, A. P., and Ranganath, C. (2007). Imaging recollection and familiarity in the medial temporal lobe: a three-component model. Trends in Cognitive Sciences, doi:10.1016/j.tics.2007.08.001.

Dijkstra, E. W. (1959). A note on two problems in connexion with graphs. Numerische Mathematik, 1:269-271.

Droit-Volet, S. and Wearden, J. H. (2015). Experience sampling methodology reveals similarities in the experience of time in young and elderly adults. Acta Psychologica, 156:77 - 82.

Ekstrom, A. D., Kahana, M. J., Caplan, J. B., Fields, T. A., Isham, E. A., Newman, E. L., and Fried, I. (2003). Cellular networks underlying human spatial navigation. Nature, 425:184-187.

Estes, W. K. (1955). Statistical theory of spontaneous recovery and regression. Psychological Review, 62:145-154.

Ezzyat, Y. and Davachi, L. (2011). What constitutes an episode in episodic memory? Psychological Science, 22(2):243-252.

Folkerts, S., Rutishauser, U., and Howard, M. W. (2018). Human episodic memory retrieval is accompanied by a neural contiguity effect. Journal of Neuroscience, doi.org/10.1523/JNEUROSCI.2312-17.2018.

Fries, P. (2005). A mechanism for cognitive dynamics: neuronal communication through neuronal coherence. Trends Cogn Sci, 9(10):474-480.

Fuhs, M. and Touretzky, D. (2006). A Spin Glass Model of Path Integration in Rat Medial Entorhinal Cortex. Journal of Neuroscience, 26(16):4266-4276.

Fyhn, M., Molden, S., Witter, M., Moser, E., and Moser, M. (2004). Spatial representation in the entorhinal cortex. Science, 305:1258-1264. 
Gershman, S. J., Moore, C. D., Todd, M. T., Norman, K. A., and Sederberg, P. B. (2012). The successor representation and temporal context. Neural Computation, 24(6):1553-1568.

Gershman, S. J., Schapiro, A. C., Hupbach, A., and Norman, K. A. (2013). Neural context reinstatement predicts memory misattribution. Journal of Neuroscience, 33(20):8590 - 8595.

Hafting, T., Fyhn, M., Molden, S., Moser, M.-B., and Moser, E. I. (2005). Microstructure of a spatial map in the entorhinal cortex. Nature, 436:801-806.

Hasson, U., Yang, E., Vallines, I., Heeger, D. J., and Rubin, N. (2008). A hierarchy of temporal receptive windows in human cortex. Journal of Neuroscience, 28(10):2539-2550.

Haxby, J. V., Gobbini, M. I., Furey, M. L., Ishai, A., Schouten, J. L., and Pietrini, P. (2001). Distributed and overlapping representations of faces and objects in ventral temporal cortex. Science, 293:2425-2430.

Healey, M. K. and Kahana, M. J. (2014). Is memory search governed by universal principles or idiosyncratic strategies? Journal of Experimental Psychology: General, 143(2):575-596.

Heraclitus (535 BCE - $475 \mathrm{BCE})$. You cannot step twice into the same river; for other waters are continually flowing in. Fragment 41; Quoted by Plato in Cratylus.

Heusser, A. C., Fitzpatrick, P. C., and Manning, J. R. (2018a). How is experience transformed into memory? bioRxiv, 409987.

Heusser, A. C., Ziman, K., Owen, L. L. W., and Manning, J. R. (2018b). HyperTools: a Python toolbox for gaining geometric insights into high-dimensional data. Journal of Machine Learning Research, 18(152):1-6.

Hodgkin, A. L. and Huxley, A. F. (1952). A quantitative description of membrane current and its application to conduction and excitation in nerve. Journal of Physiology, 117:500-544. 
Honey, C. J., Thesen, T., Donner, T. H., Silbert, L. J., Carlson, C. E., Devinsky, O., Doyle, J. C., Rubin, N., Heeger, D. J., and Hasson, U. (2012a). Slow cortical dynamics and the accumulation of information over long timescales. Neuron, 76:423-434.

Honey, C. J., Thomson, C. R., Lerner, Y., and Hasson, U. (2012b). Not lost in translation: Neural responses shared across languages. Journal of Neuroscience, 32(44):15277-15283.

Howard, M. W. and Eichenbaum, H. (2013). The hippocampus, time, and memory across scales. Journal of Experimental Psychology: General, page In press.

Howard, M. W. and Eichenbaum, H. (2014). Time and space in the hippocampus. Brain Research, $1621: 345-354$.

Howard, M. W. and Kahana, M. J. (2002). A distributed representation of temporal context. Journal of Mathematical Psychology, 46:269-299.

Howard, M. W., MacDonald, C. J., Tiganj, Z., Shankar, K. H., Du, Q., Hasselmo, M. E., and H., E. (2014). A unified mathematical framework for coding time, space, and sequences in the medial temporal lobe. Journal of Neuroscience, 34(13):4692-4707.

Howard, M. W., Viskontas, I. V., Shankar, K. H., and Fried, I. (2012). Ensembles of human MTL neurons "jump back in time" in response to a repeated stimulus. Hippocampus, 22:1833-1847.

Huth, A. G., de Heer, W. A., Griffiths, T. L., Theunissen, F. E., and Gallant, J. L. (2016). Natural speech reveals the semantic maps that tile human cerebral cortex. Nature, 532:453-458.

Huth, A. G., Nisimoto, S., Vu, A. T., and Gallant, J. L. (2012). A continuous semantic space describes the representation of thousands of object and action categories across the human brain. Neuron, 76(6):1210-1224. 
Jacobs, J., Weidemann, C. T., Miller, J. F., Solway, A., Burke, J. F., Wei, X., Suthana, N., Sperling, M. R., Sharan, A. D., Fried, I., and Kahana, M. J. (2013). Direct recordings of grid-like neuronal activity in human spatial navigation. Nature Neuroscience, 16:1188-1190.

Joubert, C. E. (1990). Subjective expectations of the acceleration of time with aging. Perceptual and Motor Skills, 70:334.

Kahana, M. J. (1996). Associative retrieval processes in free recall. Memory \& Cognition, 24:103109.

Kelley, W. M., Macrae, C. N., Wyland, C. L., Caglar, S., Inati, S., and Heatherton, T. F. (2002). Finding the self? an event-related fmri study. Journal of Cognitive Neuroscience, 14(5):785794.

Kjelstrup, K., Solstad, T., Brun, V., Hafting, T., Leutgeb, S., Witter, M., Moser, E., and Moser, M. (2008). Finite scale of spatial representation in the hippocampus. Science, 321(5885):140-143.

Landauer, T. K. and Dumais, S. T. (1997). A solution to Plato's problem: the latent semantic analysis theory of acquisition, induction, and representation of knowledge. Psychological Review, 104:211-240.

Lemlich, R. (1975). Subjective acceleration of time with aging. Perceptual and Motor Skills, $41: 235-238$.

Lerner, Y., Honey, C. J., Silbert, L. J., and Hasson, U. (2011). Topographic mapping of a hierarchy of temporal receptive windows using a narrated story. Journal of Neuroscience, 31(8):29062915.

Long, N. M., Danoff, M. S., and Kahana, M. J. (2015). Recall dynamics reveal the retrieval of emotional context. Psychonomic Bulletin \& Review, 22:1328-1333. 
MacDonald, C., Lepage, K., Eden, U., and Eichenbaum, H. (2011). Hippocampal "time cells" bridge the gap in memory for discontiguous events. Neuron, 71(4):737-749.

Manning, J. R. (2019). Episodic memory: mental time travel or a quantum 'memory wave' function? PsyArXiv, doi:10.31234/osf.io/6zjwb.

Manning, J. R., Hulbert, J. C., Williams, J., Piloto, L., Sahakyan, L., and Norman, K. A. (2016). A neural signature of contextually mediated intentional forgetting. Psychonomic Bulletin and Review, 23(5):1534-1542.

Manning, J. R. and Kahana, M. J. (2012). Interpreting semantic clustering effects in free recall. Memory, 20(5):511-517.

Manning, J. R., Lew, T. F., Li, N., Sekuler, R., and Kahana, M. J. (2014). MAGELLAN: a cognitive map-based model of human wayfinding. Journal of Experimental Psychology: General, 143(3):1314-1330.

Manning, J. R., Norman, K. A., and Kahana, M. J. (2015). The role of context in episodic memory. In Gazzaniga, M., editor, The Cognitive Neurosciences, Fifth edition, pages 557-566. MIT Press.

Manning, J. R., Polyn, S. M., Baltuch, G., Litt, B., and Kahana, M. J. (2011). Oscillatory patterns in temporal lobe reveal context reinstatement during memory search. Proceedings of the National Academy of Sciences, USA, 108(31):12893-12897.

Manning, J. R., Sperling, M. R., Sharan, A., Rosenberg, E. A., and Kahana, M. J. (2012). Spontaneously reactivated patterns in frontal and temporal lobe predict semantic clustering during memory search. The Journal of Neuroscience, 32(26):8871-8878.

Manns, J. R., Howard, M. W., and Eichenbaum, H. (2007). Gradual changes in hippocampal activity support remembering the order of events. Neuron, 56(3):530-540. 
Mau, W., Sullivan, D. W., Kinsky, N. R., Hasselmo, M. E., Howard, M. W., and Eichenbaum, H. (2018). The same hippocampal CA1 population simultaneously codes temporal information over multiple timescales. Current Biology, 28(1):1499-1508.

McClelland, J. L., Rumelhart, D. E., and PDP Research Group (1986). Parallel distributed processing. Explorations in the Microstructure of Cognition, 2:216-271.

McKenzie, S. and Buzsáki, G. (2016). Micro-, Meso- and Macro-Dynamics of the Brain, Research and Perspectives in Neurosciences, chapter Hippocampal mechanisms for the segmentation of space by goals and boundaries. Springer.

McNaughton, B. L., Battaglia, F. P., Jensen, O., Moser, E. I., and Moser, M.-B. (2006). Path integration and the neural basis of the 'cognitive map'. Nature Reviews Neuroscience, 7:663678.

Mikolov, T., Chen, K., Corrado, G., and Dean, J. (2013). Efficient estimation of word representations in vector space. arXiv, 1301.3781.

Miller, G. (1995). WordNet: a lexical database for English. Communications of the ACM, $38(11): 41$.

Miller, J. F., Lazarus, E., Polyn, S. M., and Kahana, M. J. (2013a). Spatial clustering during memory search. Journal of Experimental Psychology: Learning, Memory and Cognition, 39(3):773781.

Miller, J. F., Neufang, M., Solway, A., Brandt, A., Trippel, M., Mader, I., Hefft, S., Merkow, M., Polyn, S. M., Jacobs, J., Kahana, M. J., and Schulze-Bonhage, A. (2013b). Neural activity in human hippocampal formation reveals the spatial context of retrieved memories. Science, 342(6162):1111-1114. 
Mitchell, T., Shinkareva, S., Carlson, A., Chang, K., Malave, V., Mason, R., and Just, M. (2008). Predicting human brain activity associated with the meanings of nouns. Science, 320(5880):1191.

Momennejad, I., Russek, E. M., Cheong, J. H., Botvinick, M. M., Daw, N. D., and Gershman, S. J. (2017). The successor representation in human reinforcement learning. Nature Human Behavior, 1:680-692.

Montchal, M. E., Reagh, Z. M., and Yassa, M. A. (2019). Precise temporal memories are supported by the lateral entorhinal cortex in humans. Nature Neuroscience, doi.org/10.1038/s41593-0180303-1.

Moser, E., Kropff, E., and Moser, M. (2008). Place cells, grid cells, and the brain's spatial representation system. Апnu Rev Neurosci, 31:69-89.

Moser, M.-B., Rowland, D. C., and Moser, E. I. (2015). Place cells, grid cells, and memory. Cold Spring Harbor Perspectives in Biology, 7:a021808.

Norman, K. A., Newman, E., Detre, G., and Polyn, S. M. (2006). How inhibitory oscillations can train neural networks and punish competitors. Neural Computation, 18:1577-1610.

O'Keefe, J. and Burgess, N. (2005). Dual phase and rate coding in hippocampal place cells: theoretical significance and relationship to entorhinal grid cells. Hippocampus, 15(7):853-866.

O'Keefe, J. and Dostrovsky, J. (1971). The hippocampus as a spatial map: Preliminary evidence from unit activity in the freely-moving rat. Brain Research, 34:171-175.

Pastalkova, E., Itskov, V., Amarasingham, A., and Buzsáki, G. (2008). Internally generated cell assembly sequences in the rat hippocampus. Science, 321:1322 - 1327.

Polyn, S. M., Natu, V. S., Cohen, J. D., and Norman, K. A. (2005). Category-specific cortical activity precedes retrieval during memory search. Science, 310:1963-1966. 
Polyn, S. M., Norman, K. A., and Kahana, M. J. (2009). A context maintenance and retrieval model of organizational processes in free recall. Psychological Review, 116(1):129-156.

Post, E. L. (1930). Generalized differentiation. Transactions of the American Mathematical Society, 32:723-781.

Radvansky, G. A. and Copeland, D. E. (2006). Walking through doorways causes forgetting: situation models and experienced space. Memory \& Cognition, 34(5):1150 - 1156.

Ranganath, C. (2018). Time, memory, and the legacy of Howard Eichenbaum. PsyArXiv, 10.31234/osf.io/9tcrp.

Ranganath, C. and Ritchey, M. (2012). Two cortical systems for memory-guided behavior. Nature Reviews Neuroscience, 13:713- 726.

Reynolds, J. R., Zacks, J. M., and Braver, T. S. (2007). A computational model of event segmentation from perceptual prediction. Cognitive Science, 31:613-643.

Robin, J. and Olsen, R. K. (2019). Scenes facilitate associative memory and integration. Learning and Memory, doi:10.1101/lm.049486.119.

Roy, M., Shohamy, D., and Wager, T. D. (2012). Ventromedial prefrontal-subcortical systems and the generation of affective meaning. Trends in Cognitive Sciences, 16(3):147-156.

Rumelhart, D., McClelland, J., and the PDP Research Group (1986). Parallel distributed processing. MIT Press.

Sahakyan, L., Delaney, P. F., Foster, N. L., and Abushanab, B. (2013). List-method directed forgetting in cognitive and clinical research: a theoretical and methodological review. Psychology of Learning and Motivation, 59:131-189. 
Sahakyan, L. and Kelley, C. M. (2002). A contextual change account of the directed forgetting effect. Journal of Experimental Psychology: Learning, Memory, and Cognition, 28(6):10641072.

Sahakyan, L. and Smith, J. R. (2014). A long time ago, in a context far, far away: Retrospective time estimates and internal context change. Journal Experimental Psychology: Learning, Memory and Cogntion, 40(1):86-93.

Sargolini, F., Fyhn, M., Hafting, T., McNaughton, B., Witter, M., Moser, M., and Moser, E. (2006). Conjunctive Representation of Position, Direction, and Velocity in Entorhinal Cortex. Science, 312(5774):758-762.

Savelli, F., Yoganarasimha, D., and Knierim, J. (2008). Influence of boundary removal on the spatial representations of the medial entorhinal cortex. Hippocampus, 18(12):1270-1282.

Schapiro, A. C., Rogers, T. T., Cordova, N. I., Turk-Browne, N. B., and Botvinick, M. M. (2013). Neural representations of events arise from temporal community structure. Nature Neuroscience, 16:486-492.

Sederberg, P. B., Howard, M. W., and Kahana, M. J. (2008). A context-based theory of recency and contiguity in free recall. Psychological Review, 115(4):893-912.

Seidenberg, M. S. and McClelland, J. L. (1989). A distributed, developmental model of word recognition and naming. Psychol Rev, 96(4):523-568.

Shankar, K. H. and Howard, M. W. (2010). Timing using temporal context. Brain Research, 1365(3-17).

Shankar, K. H. and Howard, M. W. (2012). A scale-invariant internal representation of time. Neural Computation, 24:134-193. 
Shankar, K. H., Jagadisan, U. K. K., and Howard, M. W. (2009). Sequential learning using temporal context. Journal of Mathematical Psychology, 53:474 - 485.

Shapiro, M. L. (2019). Time is just a memory. Nature Neuroscience, doi.org/10.1038/s41593-0180331-x.

Shinkareva, S. V., Mason, R. A., Malave, V. L., Wang, W., Mitchell, T. M., and Just, M. A. (2008). Using fMRI brain activation to identify cognitive states associated with perception of tools and dwellings. PLoS One, e1394:1-9.

Shohamy, D. and Daw, N. D. (2015). Integrating memories to guide decisions. Current Opinion in Behavioral Sciences, 5:85-90.

Solstad, T., Boccara, C., Kropff, E., Moser, M., and Moser, E. (2008). Representation of Geometric Borders in the Entorhinal Cortex. Science, 322(5909):1865.

Solstad, T., Moser, E. I., and Einevoll, G. T. (2006). From grid cells to place cells: A mathematical model. Hippocampus, 16:1026-1031.

Swallow, K. M., Barch, D. M., Head, D., Maley, C. J., Holder, D., and Zacks, J. M. (2011). Changes in events alter how people remember recent information. Journal of Cognitive Neuroscience, 23(5):1052 - 1064.

Swallow, K. M., Zacks, J. M., and Abrams, R. A. (2009). Event boundaries in perception affect memory encoding and updating. Journal of Experimental Psychology: General, 138(2):236 257.

Talmi, D., Lohnas, L. J., and Daw, N. D. (2019). A retrieved context model of the emotional modulation of memory. Psychological Review, 126(4):455-485. 
Taube, J., Muller, R., and Ranck, J. (1990). Head-direction cells recorded from the postsubiculum in freely moving rats. I. Description and quantitative analysis. Journal of Neuroscience, $10(2): 420-435$.

Tolman, E. C. (1948). Cognitive maps in rats and men. Psychology Review, 55:189-208.

Tsao, A., Sugar, J., Lu, L., Wang, C., Knierim, J. J., Moser, M.-B., and Moser, E. I. (2018). Integrating time from experience in the lateral entorhinal cortex. Nature, 561:57-62.

Tsitsiklis, M., Miller, J., Qasim, S. E., Inman, C. S., Gross, R. E., Willie, J. T., Smith, E. H., Sheth, S. A., Schevon, C. A., Sperling, M. R., Sharan, A., Stein, J. M., and Jacobs, J. (2019). Singleneuron representations of spatial memory targets in humans. bioRxiv, doi:10.1101/523753.

Tulving, E. (1983). Elements of Episodic Memory. Oxford, New York.

Wearden, J. H. (2015). Passage of time judgements. Consciousness and Cognition, 38:165 - 171.

Wilson, M. A. and McNaughton, B. L. (1993). Dynamics of the hippocampal ensemble code for space. Science, 261:1055-8.

Wixted, J. T. and Rohrer, D. (1994). Analyzing the dynamics of free recall: An integrative review of the empirical literature. Psychonomic Bulletin and Review, 1:89-106.

Yaffe, R. B., Kerr, M. S. D., Damera, S., Sarma, S. V., Inati, S. K., and Zaghloul, K. A. (2014). Reinstatement of distributed cortical oscillations occurs with precise spatiotemporal dynamics during successful memory retrieval. Proceedings of the National Academy of Science USA, 111(52):18727-18732.

Zacks, J. M., Speer, N. K., Swallow, K. M., Braver, T. S., and Reynolds, J. R. (2007). Event perception: a mind-brain perspective. Psychological Bulletin, 133:273-293. 
Zadbood, A., Chen, J., Leong, Y. C., Norman, K. A., and Hasson, U. (2017). How we transmit memories to other brains: Constructing shared neural representations via communication. Cereb Cortex, 27(10):4988-5000.

Zwaan, R. A. and Radvansky, G. A. (1998). Situation models in language comprehension and memory. Psychological Bulletin, 123(2):162 - 185. 